Original Research

\title{
Cerebellar transcranial direct current stimulation modulates verbal working memory
}

\author{
Andreas Boehringer ${ }^{\mathrm{a}, \mathrm{b}, *, 1}$, Katja Macher ${ }^{\mathrm{a}, 1}$, Juergen Dukart ${ }^{\mathrm{a}, \mathrm{c}}$, Arno Villringer ${ }^{\mathrm{a}, \mathrm{d}, \mathrm{e}}$, Burkhard Pleger ${ }^{\mathrm{a}, \mathrm{e}}$ \\ ${ }^{a}$ Dept. of Neurology, Max Planck Institute for Human Cognitive and Brain Sciences, Leipzig, Germany \\ ${ }^{\mathrm{b}}$ Central Institute for Mental Health, Mannheim, Germany \\ ${ }^{\mathrm{c}}$ LREN, Département des Neurosciences Cliniques, CHUV, Université de Lausanne, Lausanne, Switzerland \\ ${ }^{\mathrm{d}}$ Berlin School of Mind and Brain, Mind and Brain Institute, Berlin, Germany \\ e Dept. of Cognitive Neurology, University Hospital Leipzig, Germany
}

\section{A R T I C L E I N F O}

\section{Article history:}

Received 30 June 2012

Received in revised form

3 October 2012

Accepted 4 October 2012

Available online $\mathrm{xxx}$

\section{Keywords:}

Cerebellum

tDCS

Working memory

Digit span

\begin{abstract}
A B S T R A C T
Background: Neuroimaging studies show cerebellar activations in a wide range of cognitive tasks and patients with cerebellar lesions often present cognitive deficits suggesting a cerebellar role in higherorder cognition.

Objective: We used cathodal transcranial direct current stimulation (tDCS), known to inhibit neuronal excitability, over the cerebellum to investigate if cathodal tDCS impairs verbal working memory, an important higher-order cognitive faculty.

Method: We tested verbal working memory as measured by forward and backward digit spans in 40 healthy young participants before and after applying cathodal tDCS ( $2 \mathrm{~mA}$, stimulation duration $25 \mathrm{~min})$ to the right cerebellum using a randomized, sham-controlled, double-blind, cross-over design. In addition, we tested the effect of cerebellar tDCS on word reading, finger tapping and a visually cued sensorimotor task.

Results: In line with lower digit spans in patients with cerebellar lesions, cerebellar tDCS reduced forward digit spans and blocked the practice dependent increase in backward digit spans. No effects of tDCS on word reading, finger tapping or the visually cued sensorimotor task were found.

Conclusion: Our results support the view that the cerebellum contributes to verbal working memory as measured by forward and backward digit spans. Moreover, the induction of reversible "virtual cerebellar lesions" in healthy individuals by means of tDCS may improve our understanding of the mechanistic basis of verbal working memory deficits in patients with cerebellar lesions.
\end{abstract}

(c) 2012 Elsevier Inc. All rights reserved.

\section{Introduction}

The cerebellum has long been known for its essential role in motor control in which it contributes to motor learning, as well as the planning and regulation of movements [1]. More recently, a cerebellar involvement in higher-order cognitive tasks was recognized. Cerebellar activation is consistently found in neuroimaging studies on language, working-memory, executive functions, and emotional processing [2]. Moreover, patients with

\footnotetext{
Financial disclosure: All authors have nothing to disclose.

This work was supported by the BMBF (Bernstein Focus, State Dependencies of Learning 01GQ0975; Project 18GL4DW4 to B.P.).

* Corresponding author. Central Institute for Mental Health, Systems Neuroscience in Psychiatry, J5, Mannheim, Germany.

E-mail address: post@andreas-boehringer.de (A. Boehringer).

1 Both authors contributed equally.
}

cerebellar lesions show deficits in executive functions, language production, abstract reasoning, and verbal working-memory [3-6]. These neuroimaging and clinical findings, as well as the existence of dense anatomical connections between the cerebellum and the prefrontal cortex [7], suggest that the cerebellum plays a hitherto underestimated role in higher-order cognitive function [8,9]. However, cerebellar activations during cognitive tasks not necessarily indicate cognitive processing but may rather reflect subtle contaminations by task-related motor demands. Moreover, lesions in the cerebellum often not occur in isolation making conclusions about regional specific effects of cerebellar lesions difficult. Finally, treatments associated with cerebellar disease may themselves produce cognitive deficits. A cerebellar role in higher-level cognition is thus still controversial [10,11].

Transcranial direct current stimulation (tDCS) is a non-invasive brain-stimulation technique that is used to pass a direct current 
through the brain of subjects via surface electrodes fixed to the head. It allows manipulating the excitability of cortical neurons in humans in vivo in a polarity specific manner [12]. This increasingly popular neuroscientific technique thus offers the opportunity to investigate the behavioral consequences of regional reductions or enhancements in neuronal excitability in healthy subjects. While tDCS was most frequently applied to the cerebral cortex [13], it has recently been used to investigate cerebellar contributions to verbal working memory, an important higher-order cognitive faculty. A current neuroscientific model of VWM assumes that the cerebellum contributes to the articulatory control process and phonological storage [14-16]. Cathodal tDCS, which reduces neuronal excitability in the cerebral cortex [17], over the cerebellum improves verbal working memory as measured by the paced auditory serial subtraction task (PASST) [18]. In contrast, anodal tDCS, which increases neuronal excitability in the cerebral cortex [17], as well as cathodal tDCS impair the practice-dependent proficiency increase in verbal working memory, as measured by the Sternberg item recognition task [19]. While these results indicate that cerebellar tDCS affects verbal working memory, they also suggest that the effect itself and its direction crucially depend on the specific characteristics of the tasks and experimental protocols employed.

The digit-span task is a widely accepted measure of verbal working memory which has frequently been used in clinical and experimental settings and has become an essential part of major intelligence testing systems [20]. In line with a cerebellar role in VWM [14-16], patients with cerebellar lesions show lower digit spans than healthy controls [5]. The effect of cerebellar tDCS on digit spans has not been investigated yet, but combining tDCS with digit spans in healthy individuals may constitute an appropriate lesion model that helps to improve our understanding of the mechanistic bases of the cerebellar involvement in VWM.

Based on the well investigated inhibitory influence of cathodal tDCS on cortical excitability, we here hypothesized that cathodal tDCS over the right cerebellum reduces digit spans in healthy individuals. We tested this hypothesis in a large sample of 40 individuals, using a randomized, sham-controlled, double-blind, cross-over design and two variants of the digit span task (forward and backward digit spans) as well as several control tasks.

\section{Methods}

\section{Subjects and design}

Forty young, healthy right-handed volunteers (age $=25 \pm 3$ years, range 19-32 years, 20 females) were invited. The sham-tDCS and real-tDCS sessions were separated by at least five consecutive days. Mental and physical health was assessed by means of an interview with a physician and a short physical examination. All participants gave written informed consent and the study protocol was approved by the Ethics Committee of the Medical Faculty of the University of Leipzig. We first assessed participants' performance in forward and backward digit spans as a measure of VWM as well as several control tasks (reading of color words, a visually cued sensory-motor task, finger tapping). Afterward, cathodal or sham tDCS was applied over the right cerebellum. Following tDCS application, digit spans as well as performance on the control tasks were measured again. The order of stimulation conditions and behavioral tasks was counterbalanced across subjects.

\section{Digit span task}

We used a version of the digit span task that is part of the Wechsler Adult Intelligence Scale [20]. Sequences of digits were read by the experimenter at a rate of $1 /$ second, and participants were asked to recall the digits in forward or backward order immediately after hearing them. Starting with a sequence of two digits, the number of digits per sequence was increased by one if a participant successfully recalled a given sequence length twice. Testing ended when the participant made errors on two successive trials of a given length. Performance was expressed as the number of sequences successfully recalled divided by two.

\section{Control tasks}

\section{Reading of color words}

To investigate the effect of tDCS on reading abilities and speech production, we measured the seconds needed to read aloud a list of 42 color words as fast as possible. Reading of color words serves as a baseline measure of word reading and speech production abilities in the Golden version of the Stroop task [21], a standard task in neuropsychological testing batteries.

\section{Visually cued sensorimotor task}

In order to measure the effect of cerebellar tDCS on basic motor and attentional processes, participants worked on a 0 -back version of the n-back working memory task. This task has frequently been used in clinical and neuroscientific research [22]. The 0-back condition typically serves as a motor and attentional control condition for 2-back or 3-back conditions which are characterized by substantial working memory demands. Individuals saw sequences of letters presented serially on a computer screen and were instructed to press a button upon the appearance of a previously specified probe letter. Four blocks of 30 letters each were presented and the probe letter was indicated at the beginning of each block. Stimuli were presented for $500 \mathrm{~ms}$ with a $1500 \mathrm{~ms}$ stimulus interval. The time (in seconds) needed for a correct response to the probe letter was used as the dependent variable.

\section{Finger tapping}

Finger tapping was measured to assess the effect of cerebellar tDCS on simple motor speed. Finger tapping is included as a standard motor task in the Halstead-Reitan neuropsychological test battery [23]. Participants placed their right index finger on a lever that was attached to a counting device and were instructed to tap with the finger as quickly as possible for ten seconds. This procedure was repeated seven times. The sum of the five highest tapping rates out of seven 10 seconds trials served as the variable of interest.

\section{$t D C S$}

A direct current of $2 \mathrm{~mA}$ was applied via two saline-soaked sponge electrodes $(5 \times 5 \mathrm{~cm})$ at a density of $0.08 \mathrm{~mA} / \mathrm{cm}^{2}$ for 25 min using a commercial tDCS device (NeuroConn $\mathrm{GmbH}$, Germany). To inhibit right cerebellar excitability, the cathode was placed $2 \mathrm{~cm}$ below the inion and $1 \mathrm{~cm}$ posterior to the right mastoid process. The anode was placed over the right musculus buccinator [24]. For sham tDCS, the direct current was delivered for 30 s only and then turned off. We tested the effects of cerebellar tDCS in a double-blind design. To this end, real and sham tDCS were always applied by a researcher who was not involved in testing subjects on digit-spans or the control tasks.

\section{Statistical analysis}

Two-way analysis of variance (ANOVA) for repeated measures with the factors time point of measurement (pre vs. post real/sham tDCS) and stimulation condition (real vs. sham tDCS) was used to investigate the effect of tDCS on VWM and the control tasks. Our effect of interest was the time point of measurement by stimulation 


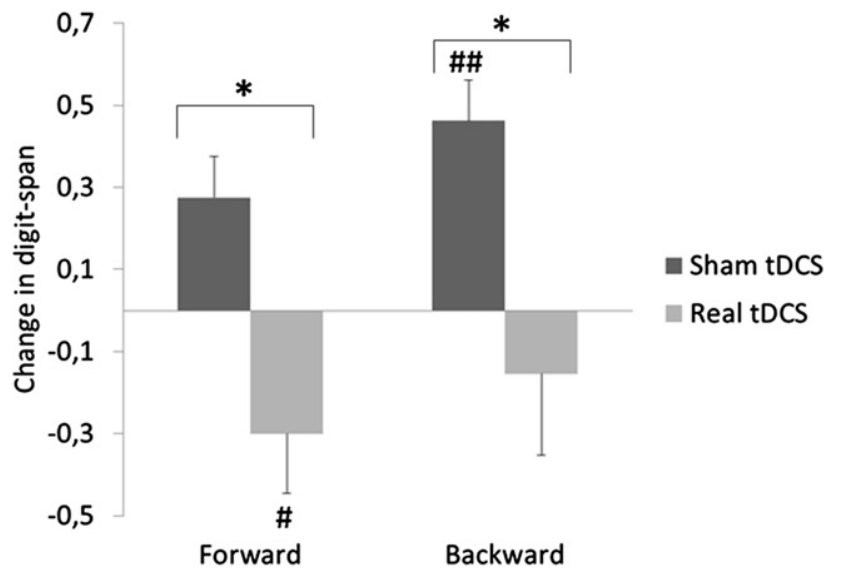

Figure 1. Mean pre to post sham/real tDCS changes in forward and backward digit spans. Real but not sham tDCS over the cerebellum significantly reduced forward digits spans. Backward digit spans increased significantly from pre to post sham tDCS measurements but remained unchanged after real tDCS over the cerebellum indicating that cerebellar tDCS blocked the practice-dependent increase in backward digit spans. $\left({ }^{*}=P<0.05\right.$, ANOVA for repeated measurements, time point of measurement (pre vs. post sham/real tDCS) by stimulation condition (sham vs. real tDCS) interaction; ${ }^{\#}=P<0.05$, paired $t$-test; ${ }^{\# \#}=P<0.001$, paired $t$-test). Forward $=$ forward digit span, Backward = backward digit span. Error-bars indicate the standard error of the mean.

condition interaction. Please note that this interaction tests whether the change in pre to post real/sham tDCS digit spans differs between the two stimulation conditions. Follow-up paired $t$-tests were used to further clarify the structure of a significant interaction.

Due to technical problems and data recording errors, data for backward digit span, reading of color words, and the visually cued serial response task were not available for one participant. Finger tapping data was not available for six participants.

\section{Results}

\section{Digit spans}

Pre sham and pre real tDCS digit spans were comparable for both measures of VWM (forward digit spans: $t_{(1,39)}=0.98$; n.s; backward digit spans: $t_{(1,38)}=0.75$; n.s.). In line with our hypothesis, ANOVA revealed a significant time point of measurement (pre vs. post sham/ real tDCS) by stimulation condition (real vs. sham tDCS; forward digit span: $F_{(1,39)}=7.43 ; P=0.010$; backward digit span: $F_{(1,38)}=4.63 ; P=0.038$ ) interaction, indicating that cathodal (i.e., inhibitory) tDCS significantly affected forward and backward digit spans (Figure 1, Table 1). Follow-up paired-t-tests showed that forward digit spans were significantly reduced after real tDCS $\left(t_{(1,39)}=2.31 ; P=0.026\right)$ but remained unchanged after sham tDCS $\left(t_{(1,39)}=-1.64\right.$; n.s.). Moreover, post real tDCS forward digit spans were significantly lower than post sham tDCS forward spans $\left(t_{(1,39)}=2.59 ; P=0.013\right)$. For backward digit spans, the number of digits correctly recalled increased after sham tDCS $\left(t_{(1,38)}=-3.46\right.$; $P=0.001)$, suggesting that repeated testing affected this measure of VWM. This increase in working memory performance was blocked after real tDCS (pre real vs. post real tDCS: $t_{(1,38)}=0.15$; n.s.). As with forward digit spans, post real tDCS backward spans were significantly lower than post sham tDCS backward spans $\left(t_{(1,38)}=2.25\right.$; $P=0.03)$.

\section{Control tasks}

Cathodal tDCS did not affect finger tapping, a simple visually cued sensorimotor task, or reading of color words (Figure 2,
Table 1

Pre and post sham/real tDCS measures of VWM, finger tapping reading, and performance in a visually cued sensorimotor task.

\begin{tabular}{|c|c|c|c|c|c|}
\hline & \multicolumn{2}{|l|}{ Sham tDCS } & \multicolumn{2}{|l|}{ Real tDCS } & \multirow[t]{2}{*}{ ANOVA } \\
\hline & Pre & Post & Pre & Post & \\
\hline $\begin{array}{l}\text { Digit span } \\
\text { forward }\end{array}$ & $6.7 \pm 0.2$ & $7.0 \pm 0.2$ & $6.9 \pm 0.1$ & $6.6 \pm 0.2$ & $F_{1,39}=7.429$ \\
\hline $\begin{array}{l}\text { Digit span } \\
\text { backward }\end{array}$ & $5.7 \pm 0.2$ & $6.2 \pm 0.2$ & $5.8 \pm 0.2$ & $5.7 \pm 0.2$ & $F_{1,38}=4.629$ \\
\hline $\begin{array}{l}\text { Reading of } \\
\text { color words } \\
\text { (s) }\end{array}$ & $13.1 \pm 0.3$ & $13.2 \pm 0.3$ & $13.2 \pm 0.3$ & $13.2 \pm 0.2$ & $F_{1,38}=0.015$ \\
\hline Finger tapping & $307.4 \pm 7.9$ & $323.2 \pm 7.1$ & $309.8 \pm 7.0$ & $325 \pm 8.7$ & $F_{1,33}=0.006$ \\
\hline $\begin{array}{l}\text { Sensorimotor } \\
\text { task (ms) }\end{array}$ & $351.4 \pm 5.1$ & $351.6 \pm 6.2$ & $354.6 \pm 7.1$ & $357.2 \pm 7.3$ & $F_{1,38}=0.250$ \\
\hline
\end{tabular}

Finger tapping: the dependent variable was the sum of the five highest counting rates out of seven $10 \mathrm{~s}$ trials. Reading of color words: the dependent variable was the time needed to read aloud a list of 42 color words. F-values represent statistical results for the time point of measurement by stimulation condition interaction as revealed by ANOVA for repeated measurements. Data presented as mean \pm standard error of the mean.

Table 1), indicating the specificity of cerebellar tDCS effects on VSTM without affecting basic motor performance or attention.

\section{Discussion}

Here, we asked whether cathodal tDCS over the cerebellum affects VWM as measured by the digit span task. In line with our hypothesis, we show that cerebellar tDCS reduces forward digit spans and blocks the practice dependent increase in backward digit spans in healthy young participants. At the same time, we did not find an effect of tDCS on finger-tapping, word-reading, and a visually cued sensorimotor task.

In good agreement with clinical work, the mean tDCS-induced reduction in forward digit-spans was moderate and of a size comparable to digit-span deficits found in patients with cerebellar lesions [5]. Our findings are also in line with evidence from a transcranial magnetic stimulation (TMS) study, showing that single puls TMS of the right cerebellum impairs VWM as measured by the Sternberg item recognition task [25]. Moreover, they support neuroimaging findings of task-related cerebellar activation during VWM [2]. Working memory has been conceptualized as a multicomponent system that consists of a central executive and several subsidiary systems [26]. In VWM, these subsidiary systems comprise an articulatory control process that is analogous to subvocal speech and a phonological store that can hold memory traces for a few seconds [26]. A current neuroscientific model of cerebellar contribution to VWM assumes that the cerebellum contributes to both of these subsidiary VWM components $[14,16,27]$. More specifically, it suggests that the superior posterior right cerebellum interacts with Broca's area and the left premotor cortex to set up and maintain a memory trace in the articulatory control process of VWM. In contrast, the posterior inferior right cerebellum is supposed to interact with left temporal-parietal cortex to contribute to phonological storage. The present finding of reduced forward digit spans after cerebellar tDCS suggests that tDCS affected these posterior cerebellar regions and their interaction with frontal and parietal cerebral areas resulting in VWM impairments as measured by the digit span task.

In contrast to the posterior cerebellar involvement in VWM and other higher-level tasks, the anterior lobe of the cerebellum contributes to sensorimotor-processing [2]. This posterior cognitive/anterior sensorimotor dichotomy in the cerebellum may explain why we failed to find an effect of cerebellar tDCS on finger tapping and a visually cued sensorimotor task, although these tasks 

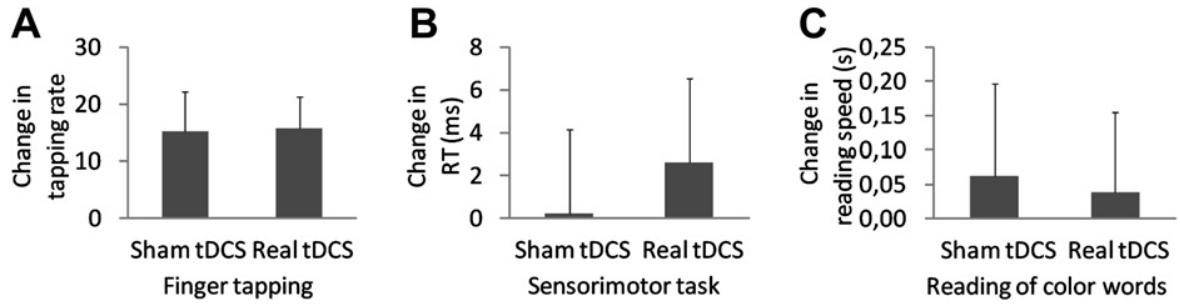

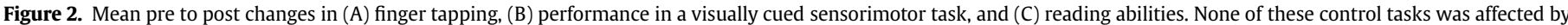

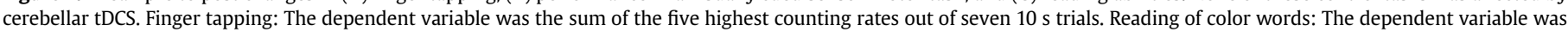

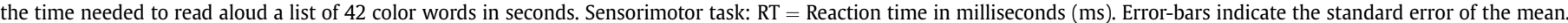

robustly activate the cerebellum in neuroimaging studies. Models of current flow during tDCS over the motor cortex suggest that a significant current density is seen only relatively local to the stimulation electrode $[28,29]$. TDCS may thus have affected the posterior lobe of the cerebellum located directly below the cathodal stimulation electrode. However, it may have failed to reach the more anterior located parts of the cerebellum involved in senorimotor processing and motor control.

Another explanation accounting for the null effect of cerebellar tDCS on our control tasks may be that these tasks were easier to perform than the relatively difficult digit span task. The tDCSinduced change in cerebellar functioning may thus have been sufficient to affect VWM but, at the same time, it may not have been strong enough to affect any of the easier control tasks. However, the control tasks used here assessed a participant's maximum performance. It thus appears unlikely that task difficulty alone or ceiling effects can account for the absence of tDCS effects on the control tasks.

TDCS over the cerebellum blocked the practice dependent increase in backward digit spans. This finding is in line with recent evidence that cerebellar tDCS impairs the practice dependent proficiency increase in the Sternberg item recognition task [19]. It is also in line with neuroimaging work showing that brain activity in the right cerebellum predicts the practice dependent proficiency increase in this VWM task [15]. In the motor domain, the cerebellum encodes internal models that reproduce the dynamic properties of body parts [30,31]. Recent models of cerebellar involvement in higher-level function assume that the cerebellum might also encode internal models that reproduce the properties of mental representations in cerebral areas connected to the cerebellum [9,31]. These internal models might allow for the skilled execution of routine cognitive operations taking place e.g. in the prefrontal cortex, a brain region crucially involved in VWM. The present finding of a tDCS induced impairment in the practice dependent increase in backward digit spans confirms the role of the cerebellum in increased task proficiency. It suggests that cerebellar tDCS affected the development of internal models in the cerebellum which contribute to the skilled execution of cognitive operations involved in verbal working memory.

In contrast to the present results, an enhancing effect of cathodal tDCS over the right cerebellum on VWM as measured by the PASST has recently been reported [18]. Our stimulation protocol was different from the one used by Pope and colleagues. Importantly, we placed the reference electrode over the right musculus buccinator while Pope and colleagues used an extracephalic location. The distance between the two electrodes influences the duration and magnitude of the induced tDCS after-effects [32]. Differences in electrode location may therefore account for some of the variability in the effects of cathodal tDCS on VWM. However, it appears unlikely that differences in electrode location alone account for an inverted influence of cathodal tDCS on VWM.
Another explanation for the diverging effects of cathodal cerebellar tDCS may rely on differences in cognitive and neural processes associated with the cognitive VWM tasks employed. During the PASST, participants listen to a series of numbers and are required to subtract the number they hear from the number immediately before it. The PASST thus involves executive processing in a different and more complex manner as the forward and backward digit span task. Importantly, Pope and colleagues found enhancing effects of cathodal tDCS only for the PASST but not an easier version of the task that requires adding (i.e., PASAT) instead of subtracting numbers, suggesting that the amount of executive processing involved by a VWM task affects the effect of cathodal tDCS on VWM. The lateral prefrontal cortex is crucially involved in cognitive control and executive processing [33] and the cerebellum functionally interacts with this brain region during VWM $[14,16,27]$. Thus, we suggest that cathodal cerebellar tDCS enhances VWM only if a certain threshold of prefrontal cortical task involvement is exceeded. Future studies will investigate this hypothesis in more detail.

The neuroscientific model by Desmond and colleagues $[14,16,27]$ postulates a specific role of the right cerebellum in VWM. Thus, we decided to apply tDCS over the right cerebellum. While neuroimaging studies support the model by Desmond and colleagues that VWM is more lateralized to the right posteriorinferior cerebellum, they furthermore identified VWM related brain activity not only in right but also left superior-posterior cerebellar areas [2]. This suggests that not only the right, but both cerebellar hemispheres contribute to VWM. In the present study we only focused on the right cerebellar hemisphere and found a tDCSinduced impairment in VWM. However, future studies should take advantage of tDCS to systematically compare the specific contributions of left and right cerebellar regions to VWM.

On the synaptic level, cathodal tDCS reduces the excitability of cortical neurons in the motor cortex [12]. In line with this cerebral effect, it reduces the inhibitory tone the cerebellum exerts on the left primary motor cortex when applied over the right cerebellum [24]. The after-effects of cathodal tDCS (which were studied here) on the motor cortex depend on the modulation of glutaminergic synapses and involve N-methyl-D-aspartate (NMDA) receptors [12]. In the cerebellum, NMDA receptors are involved in the regulation of synaptic efficacy at inhibitory synapses between interneurons and Purkinje cells [34] and presynaptic NMDA receptors play a role in cerebellar long-term depression [35]. It is tempting to speculate that cerebellar tDCS interfered with glutaminergic transmission in the cerebellum and may have affected synaptic efficacy between Purkinje cells and interneurons. This in turn may have impaired the interaction of posterior inferior and superior cerebellar regions with frontal and parietal cortical areas in the present study.

In sum, we show that cathodal cerebellar tDCS impairs VWM as measured by forward digit spans and blocks the practice dependent increase in backward digit spans. These findings support recent 
models of cerebellar involvement in higher order cognition. TCDS together with VWM tasks such as the digit span task may thus constitute an appropriate experimental setup for the investigation of the neurophysiological bases of the cerebellar involvement in VWM.

\section{References}

[1] Glickstein M, Strata P, Voogd J. Cerebellum: history. Neuroscience 2009; 162(3):549-59.

[2] Stoodley CJ, Schmahmann JD. Functional topography in the human cerebellum: a meta-analysis of neuroimaging studies. Neuroimage 2009;44(2): 489-501.

[3] Schmahmann JD, Sherman JC. The cerebellar cognitive affective syndrome. Brain 1998;121(Pt 4):561-79.

[4] Timmann D, Daum I. How consistent are cognitive impairments in patients with cerebellar disorders? Behav Neurol 2010;23(1-2):81-100.

[5] Ravizza SM, McCormick CA, Schlerf JE, Justus T, Ivry RB, Fiez JA. Cerebellar damage produces selective deficits in verbal working memory. Brain 2006; 129(Pt 2):306-20.

[6] Ben-Yehudah G, Fiez JA. Impact of cerebellar lesions on reading and phonological processing. Ann N Y Acad Sci 2008;1145:260-74.

[7] Middleton FA, Strick PL. Cerebellar projections to the prefrontal cortex of the primate. J Neurosci 2001;21(2):700-12.

[8] Ramnani N. The primate cortico-cerebellar system: anatomy and function. Nat Rev Neurosci 2006;7(7):511-22.

[9] Ito M. Control of mental activities by internal models in the cerebellum. Nat Rev Neurosci 2008;9(4):304-13.

[10] Glickstein M, Doron K. Cerebellum: connections and functions. Cerebellum 2008;7(4):589-94.

[11] Glickstein M. Thinking about the cerebellum. Brain 2006;129(Pt 2):288-90.

[12] Stagg CJ, Nitsche MA. Physiological basis of transcranial direct current stimulation. Neuroscientist 2011;17(1):37-53.

[13] Nitsche MA, Cohen LG, Wassermann EM, Priori A, Lang N, Antal A, et al. Transcranial direct current stimulation: state of the art 2008. Brain Stimul 2008; 1(3):206-23.

[14] Chen SH, Desmond JE. Temporal dynamics of cerebro-cerebellar network recruitment during a cognitive task. Neuropsychologia 2005:43(9):1227-37.

[15] Kirschen MP, Chen SH, Schraedley-Desmond P, Desmond JE. Load- and practice-dependent increases in cerebro-cerebellar activation in verbal working memory: an fMRI study. Neuroimage 2005;24(2):462-72.

[16] Chen SH, Desmond JE. Cerebrocerebellar networks during articulatory rehearsal and verbal working memory tasks. Neuroimage 2005;24(2):332-8.

[17] Nitsche MA, Paulus W. Excitability changes induced in the human motor cortex by weak transcranial direct current stimulation. J Physiol 2000;527 (Pt 3):633-9.
[18] Pope PA, Miall RC. Task-specific facilitation of cognition by cathodal transcranial direct current stimulation of the cerebellum. Brain Stimul 2012;5(2): $84-94$.

[19] Ferrucci R, Marceglia S, Vergari M, Cogiamanian F, Mrakic-Sposta S, Mameli F, et al. Cerebellar transcranial direct current stimulation impairs the practicedependent proficiency increase in working memory. J Cogn Neurosci 2008; 20(9):1687-97.

[20] Wechsler D. Wechsler adult intelligence scale-fourth edition. San Antonio: Pearson; 2008.

[21] Golden C, Freshwater S. Stroop color and word test: a manual for clinical and experimental uses. Chicago, IL: Stoelting; 2002.

[22] Owen AM, McMillan KM, Laird AR, Bullmore E. N-back working memory paradigm: a meta-analysis of normative functional neuroimaging studies. Hum Brain Mapping 2005;25(1):46-59.

[23] Reitan R, Wolfson D. The Halstead-Reitan neuropsychological test battery: theory and clinical interpretation. Neuropsychology Press; 1985.

[24] Galea JM, Jayaram G, Ajagbe L, Celnik P. Modulation of cerebellar excitability by polarity-specific noninvasive direct current stimulation. J Neurosci 2009 29(28):9115-22.

[25] Desmond JE, Chen SHA, Shieh PB. Cerebellar transcranial magnetic stimulation impairs verbal working memory. Ann Neurol 2005;58(4):553-60.

[26] Baddeley A. Working memory: looking back and looking forward. Nat Rev Neurosci 2003;4(10):829-39.

[27] Desmond JE, Gabrieli JD, Wagner AD, Ginier BL, Glover GH. Lobular patterns of cerebellar activation in verbal working-memory and finger-tapping tasks as revealed by functional MRI. J Neurosci 1997;17(24):9675-85.

[28] Miranda PC, Lomarev M, Hallett M. Modeling the current distribution during transcranial direct current stimulation. Clin Neurophysiol 2006;117(7): 1623-9.

[29] Wagner T, Fregni F, Fecteau S, Grodzinsky A, Zahn M, Pascual-Leone A. Transcranial direct current stimulation: a computer-based human model study. Neuroimage 2007;35(3):1113-24.

[30] Imamizu H, Miyauchi S, Tamada T, Sasaki Y, Takino R, Pütz B, et al. Human cerebellar activity reflecting an acquired internal model of a new tool. Nature 2000;403(6766):192-5.

[31] Imamizu H, Kawato M. Brain mechanisms for predictive control by switching internal models: implications for higher-order cognitive functions. Psychol Res 2009;73(4):527-44.

[32] Moliadze V, Antal A, Paulus W. Electrode-distance dependent after-effects of transcranial direct and random noise stimulation with extracephalic reference electrodes. Clin Neurophysiol 2010;121(12):2165-71.

[33] Petrides M. Lateral prefrontal cortex: architectonic and functional organization. Philos Trans R Soc Lond B Biolo Sci 2005;360(1456):781-95.

[34] Duguid IC, Smart TG. Retrograde activation of presynaptic NMDA receptors enhances GABA release at cerebellar interneuron-Purkinje cell synapses. Nat Neurosci 2004;7(5):525-33.

[35] Casado M, Isope P, Ascher P. Involvement of presynaptic N-methyl-D-aspartate receptors in cerebellar long-term depression. Neuron 2002;33(1): $123-30$. 